\title{
CREATING OF 3D PICTURE IN MATLAB
}

\author{
NASCAK, L[ubomir] \& KOLEDA, P[avol]
}

\begin{abstract}
This paper describes a creation of $3 D$ image in software Matlab. For creating of $3 D$ image it is necessary to capture two stereoscopic pictures of scanned area. Thereafter these pictures are synchronized by means of established synchronization points. The final $3 D$ picture is created by combination of RGB brightness components from synchronized stereoscopic pictures. By the same way, 3D movies, which scenes are shooted by special 3D camera, are created.

Key words: $3 D$ picture, stereoscopic, synchronization, $R G B$ colour
\end{abstract}

\section{INTRODUCTION}

Standard optical methods for detection of body dimensions are not often sufficient for finding of required informations about shape of this body. In some cases, it is necessary to scan the body dimensions with two cameras. If the both cameras read a scene at the same time from different angles, it will be possible to acquire the 3D body dimensions from scanned pictures. Cameras that are scanning the same scene from different angles build pictures called "stereoscopic pictures". People feel 3D world by a similar way.

\section{SEARCHING OF SYNCHRONIZATION POINTS}

Synchronization points are found in both pictures in order to whole pictures were covered. These points are created by two laser-spotters which build elliptical areas with high brightness on white background.

Searching of synchronization points will be in progress in two steps:

- searching in the left part,

- searching in the right part.

This division avoids an origination of collisions during searching of synchronization points in the picture. Both parts are searched point-to-point, whereby points with the highest brightness level represent searched points. Search conditions are as follows:

level of factor $R \in\langle 220,255\rangle$

level of factor $G \in\langle 220,255\rangle$

Searching is in progress by single rows, whereby a size of the line with white colour is being computed at its finding. Forasmuch as the marker has an elliptical shape, its middle is found as a middle of the longest line of points with high brightness.

\section{SYNCHRONIZATION OF PICTURES}

After finding of synchronization points in both pictures, location of these pictures is adjusted in order to situate corresponding synchronization points on the same position in pictures. Position adjusting of pictures is performed in two steps (White, 2004):

- $\quad$ picture adjust by slewing
- $\quad$ picture adjust by shifting

\subsection{Picture adjust by slewing}

In each picture the angle between flowline of synchronization points and $\mathrm{x}$-axis is calculated (Fig. 1). Difference between these angles $\left(\alpha_{1}\right.$ and $\left.\alpha_{2}\right)$ represents the angle $\beta$ by which first picture will be slewed counterclockwise. Angle $\beta$ is computed by relation:

$$
\beta=\alpha_{2}-\alpha_{1}
$$

$\alpha_{1}$-deflection angle of flowline of synchronization points from $x$-axis in the first picture,

$\alpha_{2}$-deflection angle of flowline of synchronization points from $x$-axis in the second picture.

In Matlab slewing of first picture is performed by help of function imrotate:

Irot = imrotate $($ I,Beta);

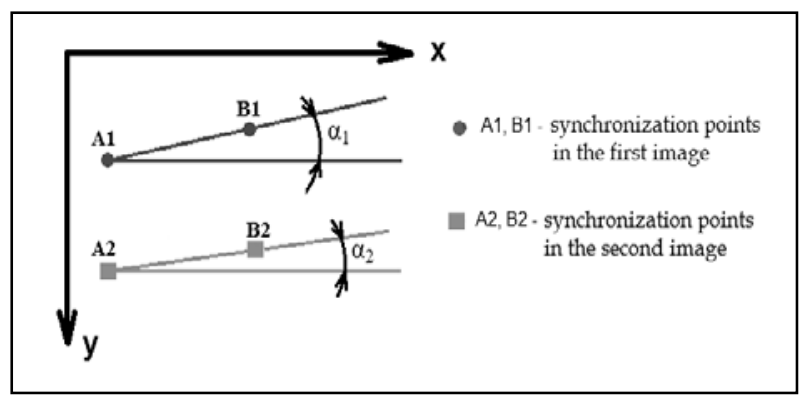

Fig. 1. Slewing of synchronization points

\subsection{Picture adjusting by shifting}

If the position of first synchronization point in the first picture is different than the position of first synchronization point in the second picture, deviations of positions these points on particular axis (DeltaX, DeltaY in Fig. 2) will be computed. Thereafter first image is shifted on particular axis about corresponding deviation.

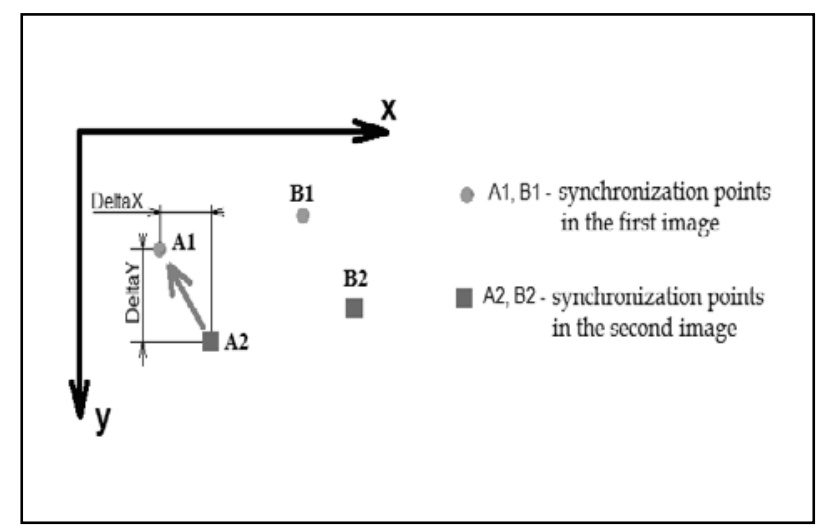

Fig. 2. Displacement of synchronization points 
In Matlab shifting of first image is performed by function imtransform (Hunt \& Lipsman \& Rosenberg, 2001):

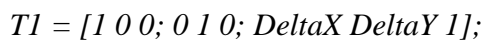

tform = maketform('affine', T1);

I1 = imtransform(I1,tform,'XData',[1 sirka],'YData',[1 vyska]);

\section{FINAL 3D PICTURE}

If pictures are synchronized, their 3-dimensional picture will be created (Fig. 3).

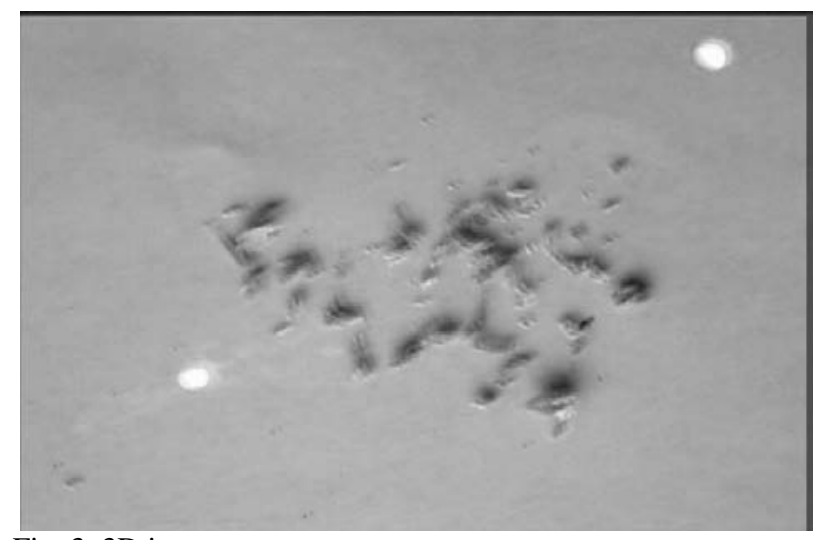

Fig. 3. 3D image

This 3-dimensional picture is created by combination of RGB brightness components of images (Fig. 4).

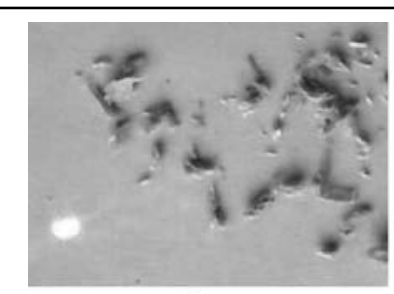

a

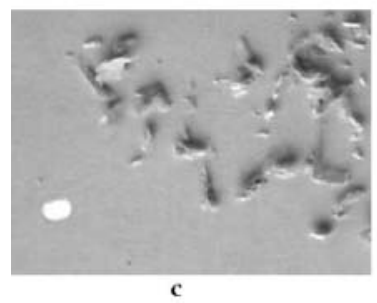

Fig. 4. Brightness components in the image: original image (a) and its factors: R (b), G (c) a B (d)

Blending of colours in software Matlab during the creation of 3D picture is performed by assumption of red colour component of the point in the first picture from the corresponding point in the second picture. This assumption of component is used on all points in the picture. Program code in Matlab is as follows (Blanchet \& Charbit, 2006):

$$
I 1(:,:, 1)=I 2(:,:, 1)
$$

Red factor of color is applied from the second picture, green and blue factor from the first picture. This combination of colours becomes evident by colour-shadows in the picture what is caused by different angles of scanning of the same scene by cameras.

Looking on this 3D picture by naked eye, a person can see the objects in it unsharply. It is necessary to watch this picture by special 3D glasses which lenses have diverse colour. 3D glasses have one lens with red colour and one with green-blue colour. These different colours of lenses causes a colour light filtration during the look trought individual lenses.

The red lens filters green and blue colour component. By the look trought this lens, tints with red colour are bright and tints with green and blue colours are dark. The second lens filters red colour component; green and blue colour components remain unchanged. Final effect of this light filtration during looking at $3 \mathrm{D}$ picture trought $3 \mathrm{D}$ glasses is that this $3 \mathrm{D}$ picture seems like cubical.

During looking at a 3D picture, the eyes converge on a point which gives absolute depth to the viewer. In the area around this point (called Panum's area), the brain merges the two images to form a single image, thus we can perceive the relative depth (Johanson, 2005).

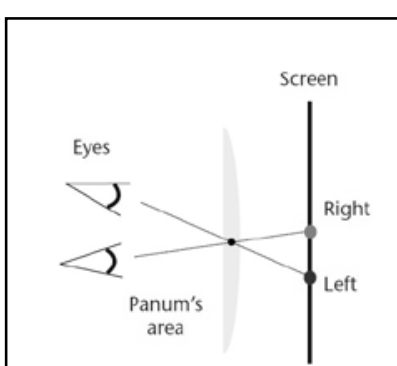

(a) (b)

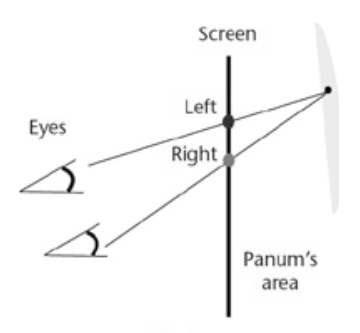

Fig. 5. Depth perception: area in front of a scene (a), area behind of a scene (Koppal \& Zitnick \& Cohen \& Kang \& Ressler \& Colburn, 2011)

\section{CONCLUSION}

By described method, it is possible to measure body dimensions in three axes. So we acquire more data about examined bodys than by common methods of body dimensions detection, where the third dimension of bodies is calculated by statistic methods. In this exploration we work with industrial cameras with image size $1600 \times 1200$ pixels, so the smallest dimension of measured elements should be bigger than $100 \mu \mathrm{m}$. By the evaluation of dimensions in 3D picture, the displacement of position of one point in both pictures is determined. This displacement is given by colour-shadows in 3D picture.

\section{REFERENCES}

Blanchet, G. \& Charbit, M. (2006). Digital Signal and Image Processing using MATLAB®, ISTE Ltd, ISBN 978-1905209-13-2, London

Hunt, B.; Lipsman, R. \& Rosenberg J. (2001). A Guide to MATLAB for Beginners and Experienced Users. Cambridge University Press. ISBN 978-0-511-07792-0

Johanson, J. (2005). Stereoscopy. Proceedings of Parallel Computing and Visualization Workshops 2005. Available from: http://www.ualberta.ca/CNS/RESEARCH/Courses/ 2005/02_Tuesday/05-Stereoscopy.pdf Accessed: 2011-0330

Koppal, S. J.; Zitnick, C. L. \& Cohen, M. F. \& Kang, S. B. \& Ressler, B. \& Colburn, A. (2011).A viewer-Centric Editor for 3D Movies. Available from: http://www.koppal.com/stereoscopy_files/stereoscopy.pdf Accessed: 2011-03-30

White, R. (2004). Computational mathematics - Models, Methods, and Analysis with MATLAB and MPI, Chapman \& Hall/CRC, ISBN 1-58488-364-2 